[0212-7199 (2004) 21: 9; pp 441-443] ANALES DE MEDICINA INTERNA Copyright @ 2004 ARAN EDICIONES, S.L.

AN. MED. InTERnA (Madrid) Vol. 21, N. ${ }^{\circ}$, pp. 441-443, 2004

\title{
Tuberculosis y cáncer. Experiencia de un hospital general
}

\author{
S. MACIÁ ESCALANTE, J. M. RAMOS RINCÓN, J. GALLEGO PLAZA, \\ A. RODRÍGUEZ LESCURE, A. SÁNCHEZ SEVILLANO, C. M. ESCOLANO \\ HORTELANO
}

Sección de Oncología Médica. Unidad de Enfermedades Infecciosas. Servicio de Medicina Interna. Hospital General Universitario de Elche. Alicante TUBERCULOSIS AND CANCER. EXPERIENCE OF A GENERAL
HOSPITAL

\section{RESUMEN}

Tuberculosis y cáncer son dos procesos que pueden aparecer interrelacionados. Se estudia la asociación entre ambas entidades.

Se han revisado de forma retrospectiva los casos diagnosticados microbiológicamente de tuberculosis en el Hospital General Universitario de Elche desde 1999 a 2002. En seis pacientes coexistía neoplasia y tuberculosis.

Se diagnosticaron 105 casos de tuberculosis, 6 de los cuales $(5,7 \%)$ tenían una neoplasia. Cuatro tuvieron tuberculosis pulmonar, uno pleuropulmonar y otro urinaria. Los dos pacientes con carcinoma broncogénico tuvieron un diagnóstico sincrónico de tuberculosis pulmonar. El diagnóstico de la neoplasia previo al de tuberculosis fue en dos casos (cáncer de mama e hipernefroma), y posterior en otros dos (cáncer de mama y de colon). La mitad de los pacientes fallecieron.

En los pacientes con neoplasia la posibilidad de coexistencia de tuberculosis no es despreciable, tanto previo al diagnóstico, concomitantemente, o tras el mismo.

PALABRAS CLAVE: Tuberculosis. Neoplasia. Tuberculosis pulmonar. Carcinoma broncogénico.

\section{ABSTRACT}

Tuberculosis and cancer are two processes in relationship. The relation between both are studied.

We analyzed retrospectively the culture-positive tuberculosis cases in from 1999 to 2002. We described the six cases with tuberculosis and cancer.

One hundred and five cases of tuberculosis were observed, 6 cases $(5,7 \%)$ had cancer. Four cases were pulmonary tuberculosis, one tuberculous pleurisy and other urinary tuberculosis urinary. The two cases with bronchogenic neoplasm had a diagnosis of tuberculosis at same time than cancer. The diagnosis of neoplasm was previous than tuberculosis in two cases (breast neoplasm and hypernephroma), and posterior in others two cases (breast and colon neoplasm). Half of theses patients died.

In patients with neoplasm, the possibility of coexist tuberculosis is not depreciable, previous, concomitant or after the diagnosis of neoplasm.

KEY WORDS: Tuberculosis. Neoplasm. Pulmonary tuberculosis. Bronchogenic neoplasm.

Maciá Escalante S, Ramos Rincón JM, Gallego Plaza J, Rodríguez Lescure A, Sánchez Sevillano A, Escolano Hortelano CM. Tuberculosis y cáncer. Experiencia de un hospital general. An Med Interna (Madrid) 2004; 21: 441-443.

\section{INTRODUCCIÓN}

La tuberculosis y el cáncer son dos procesos que están clínicamente muy relacionados. Hay situaciones en las que se sospecha una tuberculosis y finalmente se confirma una neoplasia y en otros casos ocurre a la inversa. Pero además puede existir una asociación íntima entre la tuberculosis y el cáncer; así, la tuberculosis puede preceder a un cáncer, puede aparecer sincrónicamente o acontecer tras el diagnóstico y tratamiento de la neoplasia. La asociación de tuberculosis y cáncer de pulmón es bien conocida desde hace años (1). La aparición sincrónica es poco frecuente y representa el 1-3\% de las neoplasias pulmonares (2). También se conoce, aunque con menos frecuencia, la asociación de cáncer de otra localización con tuberculosis $(3,4)$.

La asociación de neoplasia y cáncer es más frecuente en las áreas donde la prevalencia de tuberculosis es más elevada, como sucede en los países en vías de desarrollo $(1,3,4)$. En nuestro entorno, donde la prevalencia de tuberculosis es más elevada a la de otros países europeos, pero inferior a la de países en vías de desarrollo (5), la asociación de ambas entidades se ha comunicado preferentemente en forma de casos aislados $(6,7)$.

El objetivo del presente trabajo es presentar las características clínico-epidemiológicas de los pacientes con cáncer y tuberculosis en un hospital general durante 4 años.

\footnotetext{
Trabajo aceptado: 29 de marzo de 2004
} 


\section{CASOS APORTADOS}

Se han revisado de forma retrospectiva los casos de tuberculosis diagnosticados microbiológicamente en el Hospital General Universitario de Elche desde enero de 1999 a diciembre de 2002. El hospital tiene 440 camas, y cubre el área sanitaria del Baix Vinalopó en Alicante. Aquellos pacientes con neoplasia se revisaron, y se recogieron variables clínico-epidemiológicas.

Para el diagnóstico de la tuberculosis en visión directa se utilizó la tinción de auramina-rodamina. Las muestras se procesaron en los medios de Löwestein-Jensen, Colestos y en medio líquido ESP II (Difco®, Madrid) con incubación durante 60 días. La identificación definitiva de Mycobacterium tuberculosis se llevó a cabo con los procedimiento habituales. Todos los aislamientos fueron enviados al Centro Nacional de Referencia de Micobacterias de Majadahonda en Madrid para confirmar los resultados y realizar estudios de sensibilidad a los fármacos antituberculosos mediante el método de las proporciones de Canetti.

Durante el periodo de estudio se diagnosticaron 105 casos de tuberculosis, confirmada microbiológicamente, de los que $6(5,7 \%)$ tenían una neoplasia. En dos pacientes el diagnóstico de neoplasia y tuberculosis fue sincrónico; en otros dos pacientes la neoplasia precedió a la aparición de tuberculosis, y en los últimos dos se diagnosticó en primer lugar la tuberculosis y, posteriormente se le diagnosticó de neoplasia. Cuatro pacientes desarrollaron tuberculosis pulmonar, uno pleuropulmonar y otro urinaria. De los 6 pacientes, 3 fallecieron.

A continuación se describen brevemente los casos clínicos, si bien el resumen de las características clínico epidemiológicas se recogen en la tabla I.

Caso 1: Paciente varón de 55 años diagnosticado de carcinoma indiferenciado de células grandes de pulmón estadio T3N1M1 con un infiltrado en el lóbulo superior derecho. El paciente empezó con quimioterapia. Seis semanas tras el diagnóstico de neoplasia nos informan de que, en las muestras tomadas durante la broncoscopia que diagnosticó la neoplasia, crecía Mycobacterium tuberculosis. Tras conocer el resultado empezó tratamiento con isoniazida, rifampicina y pirazinamida (HRZ). Se continuó con la quimioterapia; pero el paciente falleció cinco semanas después por complicaciones de la neoplasia.
Caso 2: Paciente varón de 40 años diagnosticado de carcinoma epidermoide de traquea estadio T4, N1 M0 y un infiltrado el lóbulo superior izquierdo. Se inicia tratamiento con quimioterapia. A las cuatro semanas nos informan que en las secreciones bronquiales obtenidas durante la broncoscopia inicial crecía M. tuberculosis. Se inició tratamiento con HRZ. A las cinco semanas de tratamiento antituberculoso el paciente fallece por progresión tumoral. Tras el desenlace se informa que la cepa aislada era resistente a isoniacida.

Caso 3: Paciente varón de 66 años diagnosticado de hipernefroma del riñón derecho (estadio T2 N0 M0) del que es intervenido cinco años antes de presentar tos y dolor costal. Fue diagnósticado de una tuberculosis pleuropulmonar con un infiltrado en lóbulo superior derecho y derrame pleural derecho, para lo que recibió tratamiento con HRZ durante dos meses seguido de HR hasta completar nueve meses. Se encuentra bien 14 meses después del diagnóstico de la tuberculosis.

Caso 4: Paciente mujer de 42 años de edad diagnosticada de carcinoma ductal infiltrante de mama estadio T3N1M1. Fue tratada con quimioterapia y radioterapia. Tres años después del diagnóstico del mismo presentó tos persiste con un infiltrado cavitado en lóbulo superior derecho y fue diagnosticada de una tuberculosis pulmonar. Recibió tratamiento con HRZ dos meses e HR 4 meses, presentando buena respuesta al mismo. La enfermedad neoplásica progresó con metástasis por lo que recibió nueva quimioterapia. La paciente falleció 18 meses después de finalizar el tratamiento antituberculoso.

Caso 5: Paciente varón de 76 años diagnosticado de tuberculosis pulmonar con un infiltrado en lóbulo superior derecho, para lo que siguió tratamiento con HRZ durante 2 meses y posteriormente RH hasta completar 6 meses. A los cuatro meses del diagnóstico refería alteraciones del ritmo intestinal y persistía una discreta anemia ferropénica por lo que se solicitó una colonoscopia. En la misma se diagnóstico de un adenocarcinoma de colon de sigma, que tras ser intervenido fue estadiado en T1N0M0. El paciente fue intervenido y completó tratamiento durante 6 meses. Posteriormente ha recibido radioterapia y a continuación quimioterapia con 5 fluoracilo durante 6 meses con una buena tolerancia. El paciente esta vivo 3 años después del diagnóstico de la neoplasia.

Caso 6: Paciente de 57 años diagnosticada de tuberculosis urinaria y tratada satisfactoriamente con HRZ durante 2 meses y después

TABLA I

RESUMEN DE LOS PACIENTES CON TUBERCULOSIS Y NEOPLASIA

\begin{tabular}{|c|c|c|c|c|c|c|}
\hline & Paciente 1 & Paciente 2 & Paciente 3 & Paciente 4 & Paciente 5 & Paciente 6 \\
\hline Sexo & Varón & Varón & Varón & Mujer & Varón & Mujer \\
\hline Edad & 55 & 40 & 66 & 42 & 76 & 57 \\
\hline Localización de neoplasia & Pulmón & Pulmón & Riñón & Mama & Colon & Mama \\
\hline Tipo histológico & $\begin{array}{l}\text { Indiferenciado de } \\
\text { células grandes }\end{array}$ & Epidermoide & Hipernefroma & Ductal infiltrante & Adenocarcinoma & Lobulillar infiltrante \\
\hline $\begin{array}{l}\text { Secuencia entre el } \\
\text { diagnóstico de neoplasia* }\end{array}$ & Concomitante & Concomitante & Previo (5 años) & Previo (3 años) & Posterior (4 meses) & Posterior (14 meses) \\
\hline Tratamiento con radioterapia & No & No & No & Sí & Sí & Sí \\
\hline Baciloscopia de esputo & Negativa & Negativa & Positiva & Negativa & Negativa & No realizada \\
\hline Muestra de cultivo & $\begin{array}{l}\text { Aspirado bronquial, } \\
\text { esputo }\end{array}$ & $\begin{array}{l}\text { Aspirado bronquial, } \\
\text { esputo }\end{array}$ & $\begin{array}{l}\text { Esputo, liquido } \\
\text { pleural }\end{array}$ & Esputo & Esputo & Orina \\
\hline Tipo de tuberculosis & Pulmonar & Pulmonar & Pulmonar y pleural & Pulmonar & Pulmonar & Urinaria \\
\hline $\begin{array}{l}\text { Duración del tratamiento } \\
\text { antituberculoso }\end{array}$ & 5 semanas & 6 semanas & 36 semanas & 26 semanas & 26 semanas & 28 semanas \\
\hline
\end{tabular}

\footnotetext{
${ }^{*}$ Entre corchetes el periodo de tiempo entre el diagnostico de neoplasia y la tuberculosis o viceversa.
} 
HR 4 meses más. Un año y medio después en un prueba de cribaje de cáncer de mama fue diagnosticado de un carcinoma lobulillar de mama derecha estadio T1c N0 M0, tratada con tumorectomía, radioterapia adyuvante y hormonoterapia con una buena evolución.

\section{DISCUSIÓN}

Lo más conocido es la asociación de tuberculosis pulmonar y cáncer broncogénico; los dos tipos de cáncer más frecuentemente asociado son el epidermoide y el adenocarcinoma $(2,8)$. Los pacientes más afectados son generalmente los de edades más avanzadas $\mathrm{y}$, normalmente, fumadores; en nuestro caso los pacientes eran fumadores $(4,8)$. Se ha descrito una alta predilección del tumor por los lóbulos superiores; y tumor y tuberculosis están presentes en la misma área en el $80 \%$ de las ocasiones (1). Debido a la posible coexistencia de tuberculosis pulmonar y cáncer broncogénico, hay autores que sugieren la posibilidad de realizar broncoscopia y citología de esputo en pacientes tuberculosos con clínica atípica, y examen de esputo en pacientes con cáncer (4).

La coexistencia de neoplasia y tumor se ha visto en otras localizaciones como neoplasia de colon y tuberculosis colónica (3), neoplasia laríngea y tuberculosis laríngea (7) o neoplasia renal y tuberculosis renal (6). En nuestra serie solo coincidió la neoplasia pulmonar y el cáncer pulmonar. En los otros casos la neoplasia tuvo un origen diferente al pulmonar.

La tuberculosis pulmonar con neoplasia de otra localiza-

\section{Bibliografía}

1. Gopalakrishnan P, Miller JE, McLaughlin JS. Pulmonary tuberculosis and coexisting carcinoma: a 10 year experience and review of literature. Am Surg 1975; 41: 405-8

2. Martínez E, Aparicio J, Cordero P, Sanchís J, León M, Perpiñá M. Coexistencia de carcinoma broncogénico y tuberculosis pulmonar activa. Arch Bronchoneumol 1995; 31: 32-34.

3. Jain BK, Chandra SS, Narasimhan R, Ananthakrishnan, Mentha RB. Coexisting tuberculosis and carcinoma of colon. Aust N Z J Surg 1991; 61: 828-31.

4. Karnak D, Kayacan O, Beder S. Reactivation of pulmonary tuberculosis in malignancy. Tumori 2002; 88: 251-4.

5. WHO. Global. Tuberculosis Control. Surveillance, planing, finanacing. Genevra: WHO Report, 2002.

6. Fernández Arjona M, Ladrón Gil C, De Castro Barbosa F, Cortés Aran- ción es poco frecuente, especialmente se ha visto con cáncer de mama y linfoma (4). En esta serie tres pacientes tenían una neoplasia diferente a la pulmonar y desarrollaron tuberculosis pulmonar.

El deterioro de la inmunidad debido a los efectos locales o sistémicos del tumor por sí mismo o al tratamiento administrado (quimio o radioterapia) puede jugar un papel en la reactivación de una tuberculosis latente (4). La quimioterapia antineoplásica puede favorecer el desarrollo de tuberculosis, en pacientes con cáncer, y debería ser considerada en estos casos como una infección oportunista. Esta situación sucedió en el caso $\mathrm{n}^{\circ} 4$; en el otro paciente en el que la tuberculosis apareció después de la neoplasia, dicha neoplasia se consideró curada tras la extirpación del tumor y probablemente este condición no jugó ningún papel en la reactivación tuberculosa (caso 3).

Hay una situación especial de tuberculosis principalmente pulmonar producida tras instilaciones vesicales de BCG, lo que se a llamado BCGitis (9). No se ha observado ningún caso en esta serie primero por la escasa utilización de esta técnica en nuestro centro y segundo porque es difícil el diagnostico microbiologico de esta forma de tuberculosis y en esta serie solo se comunican casos de tuberculosis confirmada.

Como hemos visto, existe una estrecha relación entre la tuberculosis y cáncer en nuestro medio. Por ello en los pacientes con neoplasia la posibilidad de coexistencia de tuberculosis no es despreciable, tanto previo al diagnóstico, concomitantemente, o tras el mismo. Y por ello se debe mantener la alerta en nuestro entorno. guez I. Tuberculosis renal asociado con adenocarcinoma renal: asociación inusual. Actas Urol Esp 1988; 22: 794-6.

7. Plaza Mayor G, Perez Martínez C, Sierra Granon C, Rabosa GarcíaBaquero E, Fortún Abete J, et al. Tuberculosis laríngea y cáncer laríngeo. An Otorrinolaringol Ibero Am 1988; 25: 387-97.

8. Tamura A, hebisawa A, Tanaka G, Tatsuta H, Tsuboi T, Nagai H, et al. (Active pulmonary tuberculosis in patients with lung cancer). Kekkaku 1999; 74: 797-802

9. Leyes M, Salas A, Riera M, Payeras A, Pascual JA. Infiltrados pulmonares y fiebre tras la instilación intravesical de BCG. An Med Interna (Madrid) 1997; 14: 647-8.

10. De Vega T, Sarriugarte A. Tuberculosis pulmonar y cáncer de pulmón. An Med Interna (Madrid) 1997; 14: 320. 